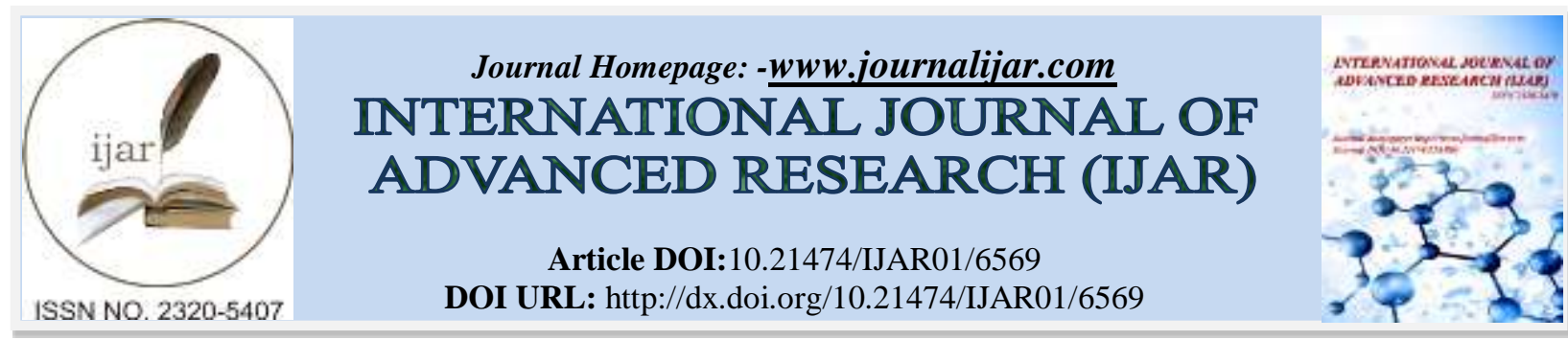

RESEARCH ARTICLE

\title{
CARACTERISATION PHENOTYPIQUE ET GENETIQUE DU RIZ AFRICAIN (ORYZA GLABERRIMA STEUD) PHENOTYPIC AND GENETIC CHARACTERIZATION OF AFRICAN RICE (ORYZA GLABERRIMA STEUD).
}

Claude Gnacadja ${ }^{1,2}$, Cecile Berthouly-Salazar ${ }^{3,4,5}$, Saïdou Nourou Sall ${ }^{6}$, Leila Zekraoui ${ }^{3}$, François Sabot ${ }^{3}$, Esther Pegalepo $^{7}$, Baboucarr Manneh ${ }^{8}$, Generose Vieira-Dalode ${ }^{2}$, Jean Moreira ${ }^{7}$, Mohamed Mansourou Soumanou ${ }^{10}$, "Paulin Azokpota ${ }^{1,2}$ and Moussa Sie?.

1. Laboratoire de Biologie Moléculaire et de Formulation Alimentaire; Ecole de Nutrition, Sciences et Technologies Alimentaires, Faculté des Sciences Agronomiques, Université Abomey Calavi Benin.

2. Laboratoire des Sciences des Aliments ; Ecole de Nutrition, Sciences et Technologies Alimentaires, Faculté des Sciences Agronomiques, Université Abomey Calavi Benin.

3. Unité Mixte de Recherche Diversité et Adaptation des Espèces, Institut de Recherche pour le Développement, Montpellier, France.

4. Laboratoire Mixte International Adaptation des Plantes et Microorganismes Associés aux Stress Environnementaux, Centre de Recherche de Bel AirDakar, Sénégal.

5. Laboratoire National de Recherches sur les Productions Végétales, Institut Sénégalais de Recherches Agricoles, Centre de Recherche de Bel AirDakar, Sénégal.

6. UFR des Sciences Agronomiques, d'Aquaculture et de Technologies Alimentaires (S2ATA), Université Gaston BERGER, Saint-Louis, Sénégal.

7. Centre du riz pour l'Afrique (AfricaRice), Abomey-Calavi, Bénin.

8. Centre du riz pour l'Afrique (AfricaRice), Sahel, Saint-Louis, Sénégal.

9. Centre du riz pour l'Afrique (AfricaRice), Antananarivo, Madagascar.

10. Unité de Recherche en Génie Enzymatique et Alimentaire (URGEA), Laboratoire d'Etude et de Recherche en Chimie Appliquée (LERCA), Département de Génie de Technologie Alimentaire, Ecole Polytechnique d'Abomey-Calavi, Université d'Abomey- Calavi Bénin.

\section{Manuscript Info}

Manuscript History

Received: 17 December 2017

Final Accepted: 19 January 2018

Published: February 2018

Keywords:-

O.glaberrima, O.sativa, locus $\mathrm{S} 1$,

African rice, diversity.

\section{Abstract}

The agronomic interest of African rice and the morphological similarities with other species, arouse the necessity to characterize African rice for recovery and conservation. The present study aims primarily to characterize phenotypically and genetically African rice for better exploitation in aid of rice producers and consumers. Indeed, the phenotypic characteristics of the rice accessions examined have been determined in two agronomics systems (upland and irrigated) carried out about 235 accessions of African rice; and afterwards, genetic characterization using a specific marker has been carried. At the end of the analyzes, with regard to the phenotypic characters, 22 O.sativa or interspecific accessions differing morphologically on several descriptors were identified. Genetically, out of 19 profiles revealed on a 935-bp band, 14 confirmed the phenotypic results. This study shows that 221 out of 235 accessions are $O$. glaberrima rice. These results show that the accessions of different species analyzed were confused during the collection. They also seems 
to validate the possibility of hybridization between the two rice species in peasant environment. The accessions characterized strengthen the conservation effort of African rice. This collection can be used for future studies, particularly with perspective to selection and running African rice with the possibility to establish a genetic model to facilitate the transfer of useful genes from O.glaberrima to O.sativa, while controlling the reproductive barrier. Thus, it will be easy to better exploit the genetic diversity of the African species of cultivated rice.

Copy Right, IJAR, 2018,. All rights reserved.

\section{Introduction:-}

Le riz est un aliment de base qui intervient fortement dans l'alimentation des populations en Afrique occidentale où près de $40 \%$ des besoins sont assurés par le marché international (Mendez del Viflar and Bauer, 2013). Deux espèces de riz sont principalement cultivées en Afrique, à savoir : Oryza sativa (le riz asiatique) etOryza glaberrima Steud (le riz africain) (Anonyme, 2002). Bien qu'en constant déclin, la production du riz africain persiste encore au nord dans le Sud-Ouest de la Mauritanie et le centre du Mali, et au sud de la côte du Golfe de Guinée en passant par la côte sénégalaise jusqu'au Sud-Ouest du Tchad (Nayar, 2012). Considéré depuis longtemps comme une source intéressante de caractères agronomiques, le riz africain a suscité bien des intérêts pour la recherche. Toutefois, son utilisation pour l'amélioration du riz asiatique a d'abord été freinée par la forte barrière reproductive qui sépare les deux espèces. Mais après plusieurs années de recherche, le gène $S 1$, principal facteur génétique impliqué dans cette barrière de stérilité, a été identifié (Sano, 1990).Ainsi,au prix d'un long effort de sélection et de croisements successifs, les premiers succès éliminant cette barrière ont été à l'origine, dans les années 2000, d'apparition de nouvelles variétés telles que le riz NERICA dont le succès a positivement impacté riz africain, négligé pendant longtemps par la recherche. Le riz africain fait partie des quelques céréales majeures avec le mil et le sorgho qui sont cultivées en Afrique. Domestiqué à partir du riz sauvage africain (O. barthii) il y a 3000 ans, soit environ 7000 ans après le riz asiatique (Huang et al., 2012), O. glaberrima provient d'une lignée évolutive complètement indépendante d'O. sativa domestiqué à partir d'O. rufipogon (Vaughan et al., 2003). Le riz africain aurait été domestiqué à partir d'O. barthii le long du fleuve Niger et aurait diffusé au niveau de deux centres secondaires de diversification: l'un le long de la côte Sénégambienne et l'autre dans les hauts plateaux intérieurs guinéens (Murray, 2004; Li et al., 2011). Le riz sauvage africain $O$. barthii aurait divergé du riz savage asiatique O. rufipogon, puis aurait diffusé vers l'Afrique conduisant à une faible proportion de diversité génétique (Vaughan et al., 2008; Nabholz et al., 2014). Alors que la diversité génétique du riz africain est a priori relativement faible, son potentiel adaptatif n'est pas négligeable comme le montre la création de vairiétés hybride NERICA (Gridley et al., 2002), dont les deux parents africains sont issus des deux groupes identifiés par Orjuela et al. (2014). Les travaux précédents ont révélé sur le plan génétique, des variétés d'O.glaberrima qui s'adaptent mieux aux contraintes locales de production et qui offrent des perspectives pour le développement des variétés inter ou intra-spécifiques productives et résistantes.Bien que moins productif que le riz asiatique, des études montrent que le riz africain possède un potentiel d'intérêt agronomique non négligeable tel que la résistance aux virus. Nwilene et Williams (2002), ont montré que des variétés de l'espèce africaine de riz présentent une bonne résistance à la cécidomyie africaine des galles.Montcho et al., (2017) ont par ailleurs rapporté que certaines accessions de $O$. glaberrima classées comme type plateau sont adaptées aux conditions de bas-fond et irrigué, et vice-versa.

Avec des intérêts agronomiques remarquables et une diversité qui serait restreinte, il devient primordial de mettre en place des programmes de valorisation et de conservation du riz africain. A ce titre, la mise en place et la caractérisation de collection de riz africain est essentielle. Or du fait des similarités morphologiques et des possibilités de croisement inter-spécifiques, il est difficile de distinguer avec certitudes les deux espèces $O$. sativa et O. glaberrima.

Depuis peu, la biologie moléculaire a permis de mettre en évidence des marqueurs génétique spécifiques qui permettent de distinguer les deux espèces (Angeles-Shim et al., 2009 ; Naoki Hirotsu et al., 2010). Le présent travail a pour but de valider l'espèce de chaque accession d'un point de vue phénotypique et génétique. Une fois validée, cette collection pourra servir à des projets de recherche futurs. 


\section{Matériel Et Method:- \\ Matériel végétal:-}

Il est constitué de 235 accessions de riz africain collectées dans les villages de la préfectureDanyi au Togo et 2 témoins (CG 14, NERICA4) ayant été tous soumis au test de germination avant essai. Ces accessions, provenant de 15 villages de Danyi (Figure 1), ont été conservées après collecte auprès des producteurs à la banque de gène de AfricaRice de Cotonou.

\section{Caractérisation phénotypiques:-}

\section{Sites d'étude:-}

L'essai en plateau a été réalisé à la ferme de la station AfricaRice à Cotonou (Bénin) tandis que l'essai en irrigué a eu lieu à la station AfricaRice Sahel de Ndiaye à Saint Louis (Sénégal).

\section{Dispositif expérimental:-}

Les essais ont été conduits dans un dispositif dénommé «augmented design in randomized complete block» $(A R C B D)$ décrit par Nokoe (2001).Ce dispositif a été utilisé en raison du nombre élevé d'accessions à tester (237) alors que la quantité de semence était limitée. Le concept de base de cette méthode est d'établir un dispositif dans lequel seuls les témoins ayant des quantités suffisantes de semences sont répétés dans chaque bloc pour servir à l'estimation de l'erreur expérimentale et de l'effet bloc (Sharma, 1988). Au total, 24 blocs de 10 entrées et deux témoins ont été constitués, soit 12 entrées par blocs. Chaque parcelle élémentaire a une densité de 42 poquets. Les distances entre poquets sont de $20 \mathrm{~cm}$ entre les lignes et $20 \mathrm{~cm}$ sur la ligne. Les parcelles élémentaires sont séparées par une allée de $30 \mathrm{~cm}$.

\section{Conduite des essais:-}

Essai en plateau:

Pour cet essai, le semis direct a été effectué à raison de 2 grains/poquet, avec un démarriage à 1plant/poquetdeux semaines après semis. Seule la fumure de fond NPK (10-18-18) est appliquée à la dose de $100 \mathrm{~kg} / \mathrm{ha}$ au semis. L'apport en eau a été fait avec un système d'arrosage et le désherbage a été manuel.

\section{Essai en irrigué:-}

Pour cet essai, les semences ont été prégermées au germoir artificiel à $30^{\circ} \mathrm{C}$ environ pendant 48 heures. Les semis sont mis dans des pots en serre. Neuf (09) jours après les semis, les pots ont été sortis des serres et exposés aux conditions naturelles pour une acclimatation. Après 48 heures, soit onze jours après semis, le repiquage en ligne a été opéré. L'herbicide sélectif LONDAX $60 \%$ (Bensulfuron-Méthyl $100 \mathrm{~g} / \mathrm{Kg}$ ) a été appliqué pour la lutte contre les mauvaises herbes avec une dose d'application recommandée de 100g/ha (AfricaRice, 2010). Cette application a ététoutefois accompagnée d'un désherbage manuel. Trois applications sucessives d'engrais $\mathrm{NP}_{2} \mathrm{O}_{5} \mathrm{KO}_{2}(150-60-60)$ ont été réalisées pour le traitement des essais. Un système d'irrigation - drainage a été mis au point pour permettre la gestion et le renouvellement de l'eau dont le niveau est controlé selon l'étape évolutive des plantes. La lutte contre les oiseaux et les rongeurs a été assurée par des filets utilisés comme couvert de tout l'espace et enfouis sous les diguettes délimitant l'espace desessais.

\section{Critères de validationphénotypique:-}

$\mathrm{Au}$ cours de ces essais, l'objectif d'étude est d'identifier sur la base des critères discriminants les variétés se distinguant de par leurs caractères phénotypiques largement distincts de l'ensemble de la collection (supposée riz africain) mise en essai. Ces observations ont été faites sur chaque site par trois observateurs indépendants suivant les normes du SES (Standard Evaluation System) et du descripteur de l'IRRI (International Rice Research Institute). Le tableau 1 présente les critères agro-morphologiques utilisés pour l'étude phénotypique

\section{Traitement des données:-}

Suivant les échelles du SES (Standard Evaluation System) et du descripteur de l'IRRI (International RiceResearch Institute), la combinaison des données issues des trois observateurs a permis de sortir pour chaque accession une moyenne des codes correspondants à chaque critère qualitatif utilisé. L'accession est validée $O$. sativa lorsqu'elle regroupe au moins quatre descripteurs spécifiques de l'espèce sur les huits considérés dans cette étude. 


\section{Caractérisation génétique:-}

Les travaux de biologie moléculaire ont été réalisés sur la plateforme de biologie moléculaire du Laboratoire Mixte International Adaptation des Plantes et Microorganismes Associés aux Stress Environnementaux (LMI LAPSE) Centre de Recherche de Bel Air à Dakar au Sénégal.

La technique d'extraction de Doyle and Doyle (1987) modifiée a été utilisée avec un traitement à la Rnase sur les extractions.

Le gène amplifié est situé sur la zone du locus $\mathrm{S} 1$ du chromosome 6. Ce locus est le plus important agissant comme une barrière reproductive entre Oryza sativa et Oryza glaberrima. Il a été cartographié sur le chromosome 6 grâce à son lien étroit avec le gène waxy (Wx) (Sano, 1990 ; Sano, 1986). Les produits PCR attendus selon l'espèce sont une bande de $935 \mathrm{pb}$ chez $O$. sativa et une bande $1384 \mathrm{pb}$ chez $O$. glaberrima. Les séquences des primers utilisés sont les suivantes

> Biodiv_10_forward: TGGGGATGCTGAATCTGATG,

> Biodiv_10_reverse: ATCTTGTTGGCAGGGTGAAC

Les conditions et le programme utilisés sont résumés dans le tableau 2.

\section{Résultats:-}

\section{Caractéristiquesphénotypiques:-}

Deux principaux indices : 1) taille de la plante et 2)cycle semis-épiaison tardif ont aidé à l'identification des accessions. La figure 2 illustre une accession (TG08-055) agro-morphologiquement différent notamment pour l'indice "Taille de la plante".

Au total 20 accessions ont été repérées par les trois observateurs en essai plateau tandis qu'en essai irrigué 22 accessions se sont distinguées (Tableau 3). L'observation du tableau 3 montre que de ces 22 accessions, 18 ont été relevées simultanément sur les deux sites d'essai ; soit un total de 24 accessions identifiées comme $O$. sativa dans au moins un essai. Deux accessions (TG08-014, TG08-46) ont été identifiées uniquement en essai plateau tandis qu'en essai irrigué spécifiquement, quatre (TG08-021, TG08-157, TG08-177 et TG08-245) ont été retenues de plus. Le croisement des résultats des deux sites montre également que les18 accessions communes aux deux sites ont été appréciées relativement dans le même sens à quelques degrés près, sur les neuf critères d'étude (Tableau 1). Par ailleurs, par un principe de comparaison aux caractères phénotypiques de l'espèce O.glaberrima, ces accessions présentent pour la plupart un mélange de caractères phénotypiques empruntés aux deux espèces.

\section{Caractéristiquesgénétiques:-}

Sur les 235 accessions testées, 188 ont été amplifiées, soit $80 \%$ de toute la collection. La révélation du polymorphisme de taille sur gel a permis d'identifier clairement 19 individus avec une bande à 935 bp soit $O$. sativa et 169 individus avec une bande à 1384 bp soit O.glaberrima (Figur 3). L'alignement des bandes étalonnées au marqueur de taille (de $1000 \mathrm{pb}$ ) révèle que les variétés $78,80,82$ et 83 présentent une bande de $935 \mathrm{pb}$ correspondant à O.sativa alors que les variétés 74,75 ou 86 et 91 présentent une bande de $1384 \mathrm{pb}$ correspondant à l'espèce O.glaberrima. L'observation de ces profils a permis de valider l'identité des variétés constituant la collection d'étude. Une analyse distinctive a permis de croiser ces résultats avec ceux obtenus à l'aide de la caractérisation agro-morphologique, spécifiquement au niveau des 24 accessions n'ayant pas présenté les caractéristiques agromorphologiques de l'espèce O.glaberrima.

Le tableau 3 présente la combinaison des résultats pour ces 24 accessions. Ce tableau montre que sur les 18 accessions identifiées dans les deux essais en tant que O.sativa 14 ont été validées et les 4 autres n'ayant pas amplifiées. Seules trois des accessions identifiés en tant que O.glaberrimaau plan phénotypique sont en réalité des O.sativa, soit un taux d'erreur de 3/212 O.glaberrima. Par ailleurs, Les TG08-021, TG08-157, TG08-177 et TG08245 qui se sont distingués uniquement en essai irrigué se révèlent au gel avec une bande de 1384pb pour TG08-021 et TG08-157 et une bande de 935pb pour TG08-177 et TG08-245.

\section{Discussion:-}

Caractéristiques phénotypiques des accessions testées:-

Toutes les accessions du matériel d'étude ont terminé leur cycle,ce qui renseigne sur leur bon pouvoir germinatif et leur vigueur végétative. Les indices de distinctions utilisés dans cette étudeont favorisé la précision dans les scores 
d'appréciation. En effet, il a été rapporté que des descripteurs agronomiques liés aux caractères "taille des plants et nombre de talles" discriminent les populations de riz (Ojo et al., 2009 ; Moukoumbi et al., 2011). De nos jours, le riz africain est rarement cultivé en culture pure. Il est souvent associé au riz asiatique qui est de plus en plus dominant sur le continent (Nayar, 2012). Toutefois, les variétés relevées de façon spécifique dans chaque site pourraient être le résultat de l'effet environnement ou des marges d'erreurs d'observations qui ne seraient pas nuls. Le mélange de traits phénotypiques observés dans les deux sites serait dû à l'influence des facteurs génétiques ou environnementaux lors de l'expression des caractères au cours du développement des plants. Le degré d'expression génétique montrant ces distinctions pourraient être le résultat d'une éventuelle introgression de gènes au cours de la domestication du riz africain et qui peut influencer à une certaine échelle les comportements agro-morphologiques. La précision relative aux degrés d'observation et au faible écart entre les scores attribués par les observateurs d'un même site sur un critère donné, résident dans le fait que non seulement le riz africain a généralement un phénotype très caractéristique de l'espèce O.glaberrima, mais aussi, la plante de riz dans sa morphologie présente des caractères précis liés à chaque étape de son cycle de développement. En effet, depuis l'époque précoloniale, Carney a fait remarquer que l'illustration du plant de riz présente des caractéristiques qui sont typiques à l'espèce africaine(Carney, 2001): d'abord, le plant du riz présente une panicule dressée, ensuite, les glumelles sont aristées, c'est-à-dire qu'il y a un prolongement de l'apex,ce qui correspond à la présence de barbes (Vido, 2011).

Ces observations basées sur des variables qualitatifs ont permis de noter une probable diversité dans la collection.Par ailleurs, une large diversité des variétés du riz africain à partir des descripteurs agro-morphologiques qualitatifs a été rapportée (Hien et al., 2007).

On retient théoriquement que ces variétés $O$. sativa ou des interspécifiques ont été enregistrées sur une marge d'erreur d'informations lors de la collecte. Il pourrait s'agir également d'un mécanisme complexe d'expression des caractères génétiques. En effet, des flux de gènes entre les deux espèces, suivant l'introduction faite par l'homme à travers l'hybridation introgressive entre les rufipogons asiatiques et les formes africaines ont été observés(Dally and Second, 1990).

\section{Caractéristiques génétiques des accessions testées:-}

Les profils génétiques observés ont permis d'identifier à une échelle donnée les individus O.sativa de notre collection d'étude. Les amorces utilisées ont amplifié le locus S1, principal facteur génétique impliqué dans la barrière de stérilité lors des croisements entre les deux espèces (Sano, 1990). Sous réserve d'élargir cette étude à une caractérisation impliquant des marqueurs à haut potentiel d'identification, 19 variétés ont présenté nettement un profil génétique de O.sativa. L'analyse tenant compte des profils observés au plan agro-morphologique montre que 14 de ces variétés se sont révélées simultanément O.sativa tantau plan agro-morphologique que génétique. Plusieurs facteurs pourraient expliquer le cas des 5 autres variétés au profil génétique de $935 \mathrm{pb}$ précédemment identifiés aux essais agronomiques comme O.glaberrima: L'introgression de gènes au cours de la domestication du riz africain peut influencer à une certaine échelle les comportements agro-morphologiques. Au total 46 variétés de la collection n'ont pu être confirmées O.glaberrima. Il s'agit en effet d'une part des variétés pour lesquelles il n'y a pas eu d'amplification au PCR (ou profil incertain dû à des amplifications non spécifiques) et d'autre part celles qui ont des profils agro-morphologique et génétique différents. Il n'est pas prudent de conclure quant à leur validation car des recherches avancées tenant compte d'autres gènes pourront révéler avec précision leur profil. Dans ce projet d'amélioration variétale impliquant les potentiels (agronomiques et de qualité grain) du riz africain, la question de levée de barrière de stérilité est au centre des essais de croisements. Un projet de génotypage des SNP permettrait de séquencer et d'étudier les gènes collatéraux qui pourraient être impliqués dans la stérilité des hybrides comme le locus S1 qui exerce l'effet le plus important sur la fertilité des hybrides (Garavito et al., 2010). Dans leur étude, Garavito et al., (2010) ont déterminé les bases génétiques de la barrière reproductive entre O.sativa et O.glaberrima, deux espèces séparées par une forte barrière reproductive, qui est le résultat de l'action cumulée de plusieurs locus qui rendent les hybrides F1 complètement male stériles et partiellement femelle stériles. Les résultats obtenus ont montré la présence d'au moins deux autres locus génétiquement liés à $\mathrm{S} 1$, et de voir des interactions épistatiques et des incompatibilités alléliques entre ces locus. Koide et al.,(2008) ont également cartographié génétiquement ce locus S1 sur le chromosome 6 en utilisant des lignées quasi isogéniques dans un intervalle de 40kb contrairement à une région longue de seulement 28 kilobases (Guyot et al., 2011) trouvée par l'équipe Génome et Développement du Riz de l'UMR DIADE (IRD) qui a aussi entrepris une étude de cartographie génétique à haute résolution du gène $S 1$ en utilisant quatre populations de rétrocroisement entre O.sativa et O.glaberrima pour la caractérisation de leurs fréquences alléliques grâces aux marqueurs SSR et R-BIP. Au cours du présent travail, les échantillons non amplifiés ont l'attention et ont fait objet deplusieurs reprises d'extraction d'ADN et de PCR, avec optimisation des 
paramètres. Cependant les résultats non concluants ont toujours été observés. Des études antérieures ont tout de même montré un déséquilibre de génome dû aux pertes de gènes au cours de l'histoire évolutive des espèces de riz. Une étude de correspondance génétique entre les deux espèces a montré qu'environ $7 \%$ du génome du riz africain n'a pu être mis en correspondance avec le génome du japonica; suggérant ainsi qu'un nombre important de gènes a été perdu dans la lignée de riz japonica. Cependant, cette même étude a montré que les parents proches du japonica possèdent encore les homologues de ces gènes. Il a été donc estimé qu'au cours de son évolution, O.sativa a perdu au moins un millier de gènes qui sont encore conservés dans les génomes de O.glaberrima(Hiroaki Sakai et al. 2010).

Les variétés classiques identifiées au niveau du tableau 3 feront alors objet d'une étude approfondie de caractérisation génétique afin d'identifier avec précision leur profil génétique, car même au plan agromorphologique, certaines de ces variétés ont présenté un mélange de caractères spécifiques aux deux espèces. Il est important, au vue de cette analyse, de poursuivre les travaux sur le rôle des allèles liés au S1 de Oryza glaberrima dans la levée de stérilité de l'hybride interspécifique entre O.sativa et O.glaberrima. Desessais effectués par Xianneng et al., (2010) ont montré que l'introgression grâce à la technique assistée par marqueurs de l'allèle S1-g de O.glaberrima utilisé comme parent mâle, dans des lignées de O.sativa ont levé considérablement la barrière reproductive.

Ces résultats de validation génétique permettent tout de même de sélectionner plus de $70 \%$ des variétés de la collection d'étude comme appartenant à l'espèce O.glaberrima.Il est nécessaire d'étendre cette étude, non seulement à la diversité génétique, mais aussi à l'identification des polymorphismes autour du locus $\mathrm{S} 1$ car la stérilité hybride entrave fortement le transfert de caractères favorables (Taits, gènes) de O.glaberrima à O.sativa (Xianneng et al. 2010).

Face à la demande mondiale croissante de cultures vivrières, avec la recherche de nouvelles espèces performantes, l'amélioration génétique des céréales est une approche prometteuse (Tanksley et al., 1997); car les analyses séquentielles du génome approfondissent la compréhension des caractéristiques des espèces apparentées au niveau moléculaire (Paterson et al. 2009).

\section{Conclusion:-}

Ce travail sur la caractérisation du riz africain s'est révélé comme un indice qui justifie les phénomènes d'introgression de gènes entre les deux espèces de riz au cours de leur évolution en Afrique. Les variétés exprimant un mélange de caractères génétiques ont été identifiées, de même que le profil des variétés de la collection. Cette identification pourra aider à établir un modèle génétique visant à faciliter le transfert de gènes utiles de O.glaberrima vers O.sativa, tout en maîtrisant la barrière reproductive. Ainsi, il sera aisé de mieux exploiter la diversité génétique de l'espèce africaine de riz cultivé. En perspective, l'établissement d'un modèle d'élimination gamétique (en conservant les gènes liés à d'autres caractères intéressants) dans l'hybride F1 développé par Garavito et al.,(2010) devra être approfondie et couplée à l'analyse du fonctionnement des facteurs génétiques liés à la stérilité et des phénomènes de divergence de séquence entre les deux espèces.

Remerciements: AfricaRice et projet HAAGRIM/INTRA ACP-UE pour le soutien financier et technique.

Tableau 1:- Liste des critères agro-morphologiques utilisés pour l'étude phénotypique

\begin{tabular}{|l|l|l|l|}
\hline $\mathrm{N}^{\circ}$ & Critères d'observation (Codes) & Phénotypes attendus \\
\cline { 3 - 4 } & & Pour O. glaberrima & Pour O. sativa \\
\hline 1 & Taille des ligules (TLg) & Ligule courte, tronquée, ovale & $\begin{array}{l}\text { Ligule longue, bifide, } \\
\text { allongée }\end{array}$ \\
\hline 2 & Excertion paniculaire (ExP) & Plus ou moins bonne & Moins bonne \\
\hline 3 & Aspect des feuilles, Pubescence (AsF) & Glabres & Rugueux \\
\hline 4 & Aspect des ramifications (AsR) & Nombreuses et ouvertes & $\begin{array}{l}\text { Moins nombreuses et } \\
\text { dressées }\end{array}$ \\
\hline 5 & Port paniculaire (PP) & Dressé & Les bouts penchés \\
\hline
\end{tabular}




\begin{tabular}{|l|l|l|l|}
\hline 6 & Résistance à la verse (RV) & Moins bonne & Bonne résistance \\
\hline 7 & Robustesse des tiges (RT) & Bien robuste & Moins robuste \\
\hline 8 & Couleur et forme des grains (C/F.G) & $\begin{array}{l}\text { Brun foncé, plus ou moins arrondi } \\
\text { et souvent aristé }\end{array}$ & $\begin{array}{l}\text { Brun clair (plus ou moins } \\
\text { blanc et allongé }\end{array}$ \\
\hline 9 & Homogénéité de la plante (HP) & Détermine le mélange ou non des grains semés \\
\hline
\end{tabular}

Tableau 2:- Composition du volume réactionnel : 10uL ( $\mathrm{mix}+\mathrm{ADN})$ et conditions de PCR.

Solutions (Vol par tube)

Tampon 5X Promega (2uL)

DNTP $2 \mathrm{mM}(1 \mathrm{uL})$

Amorce F $10 \mathrm{mM}(0,5 \mathrm{uL})$

Amorce R $10 \mathrm{mM}(0,5 \mathrm{ul})$

Taq Polymérase Promega $5 \mathrm{U} / \mu \mathrm{l}(0,2 \mu \mathrm{l})$

AND à 25ng $(1 \mu \mathrm{l})$

$\operatorname{Eau}(4,8 \mu \mathrm{l})$

Tableau 3:- Les variétés identifiées comme O.sativa au plan agronomique et génétique

\begin{tabular}{|c|c|c|c|}
\hline Identifiants & Agromorphologie plateau & Agromorphologie irrigué & Validation génétique \\
\hline TG08-003 & sativa & sativa & sativa \\
\hline TG08-007 & sativa & sativa & sativa \\
\hline TG08-013 & sativa & sativa & sativa \\
\hline TG08-014 & sativa & glaberrima & $N A$ \\
\hline TG08-15 & glaberrima & glaberrima & sativa \\
\hline TG08-021 & glaberrima & sativa & glaberrima \\
\hline TG08-039 & sativa & sativa & $N A$ \\
\hline TG08-046 & sativa & glaberrima & $N A$ \\
\hline TG08-48 & glaberrima & glaberrima & sativa \\
\hline TG08-055 & sativa & sativa & sativa \\
\hline TG08-61 & glaberrima & glaberrima & sativa \\
\hline TG08-074 & sativa & sativa & sativa \\
\hline TG08-078 & sativa & sativa & sativa \\
\hline TG08-083 & sativa & sativa & $N A$ \\
\hline TG08-133 & sativa & sativa & sativa \\
\hline TG08-136 & sativa & sativa & sativa \\
\hline TG08-139 & sativa & sativa & sativa \\
\hline TG08-140 & sativa & sativa & sativa \\
\hline TG08-142 & sativa & sativa & sativa \\
\hline TG08-144 & sativa & sativa & sativa \\
\hline TG08-157 & glaberrima & sativa & glaberrima \\
\hline TG08-177 & glaberrima & sativa & sativa \\
\hline TG08-180 & sativa & sativa & $N A$ \\
\hline TG08-235 & sativa & sativa & sativa \\
\hline TG08-240 & sativa & sativa & sativa \\
\hline TG08-245 & glaberrima & sativa & sativa \\
\hline TG08-248 & sativa & sativa & $N A$ \\
\hline
\end{tabular}

" NA" : profil non identifié ou absence d'amplification
Programme du Thermocycleur

- Dénaturation initiale: $94^{\circ} \mathrm{C} / 06 \mathrm{mn}$

- Pour un cycle : $94^{\circ} \mathrm{C} / 30 \mathrm{~s} ; 60^{\circ} \mathrm{C} / 45 \mathrm{~s}$; $72^{\circ} \mathrm{C} / 1 \mathrm{mn} 30 \mathrm{~s}$

- $\quad$ Elongation finale : $72^{\circ} \mathrm{C} / 10 \mathrm{mn}$

- Au total : 40 cycles

- Conservation : $10^{\circ} \mathrm{C}$ 


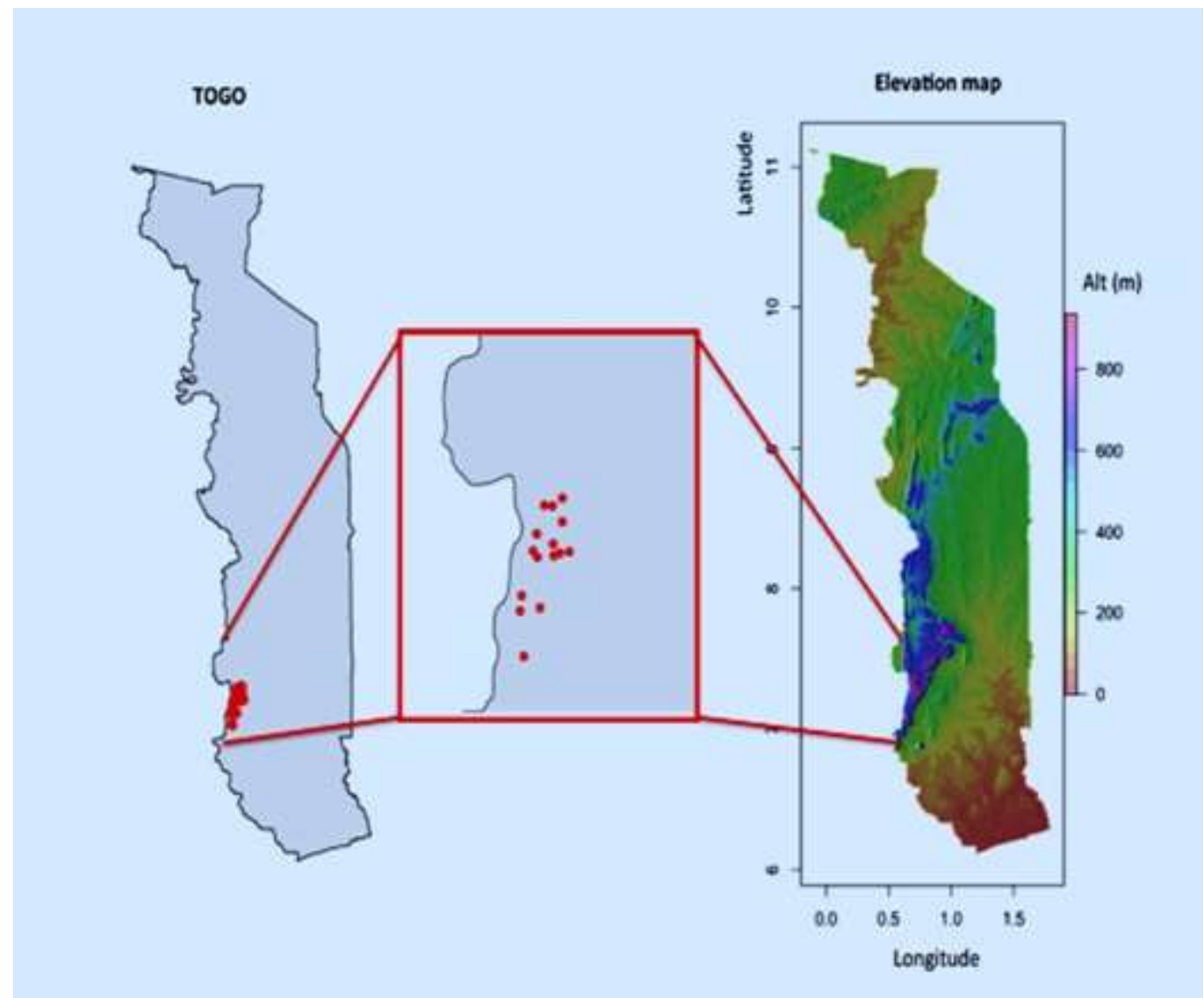

Figure 1:- Cartographie de la zone de provenance des accessions (les points désignent les villages de provenance)

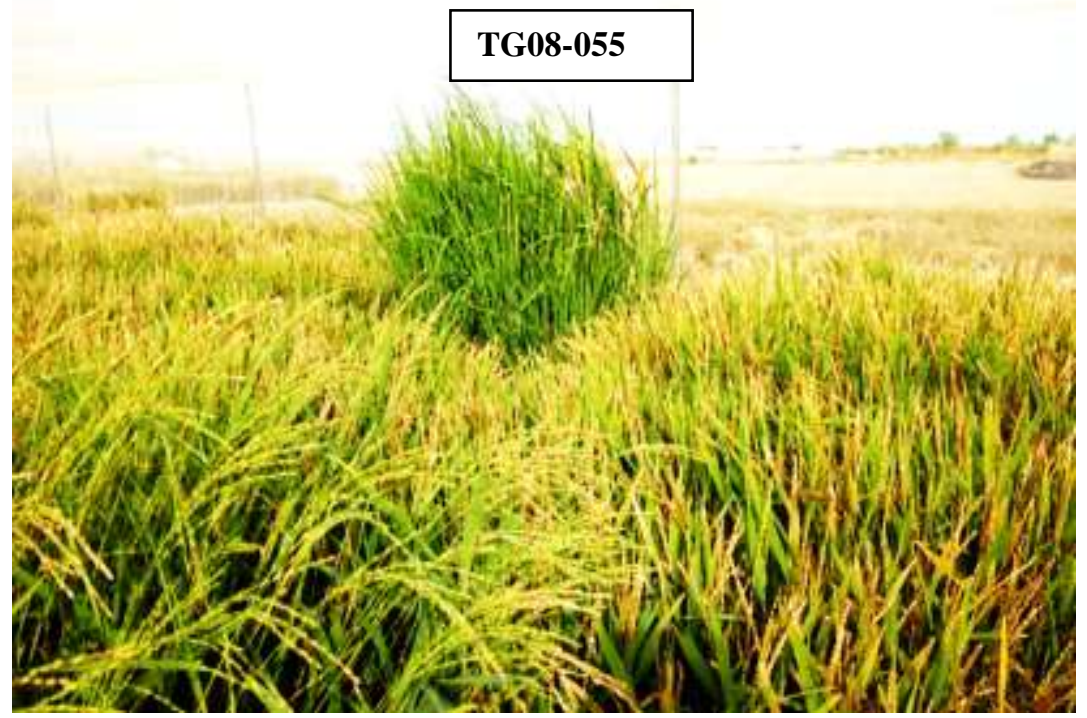

Figure 2:- Une accession (TG08-055) agro-morphologiquement différent notamment pour l'indice "Taille de la plante". 


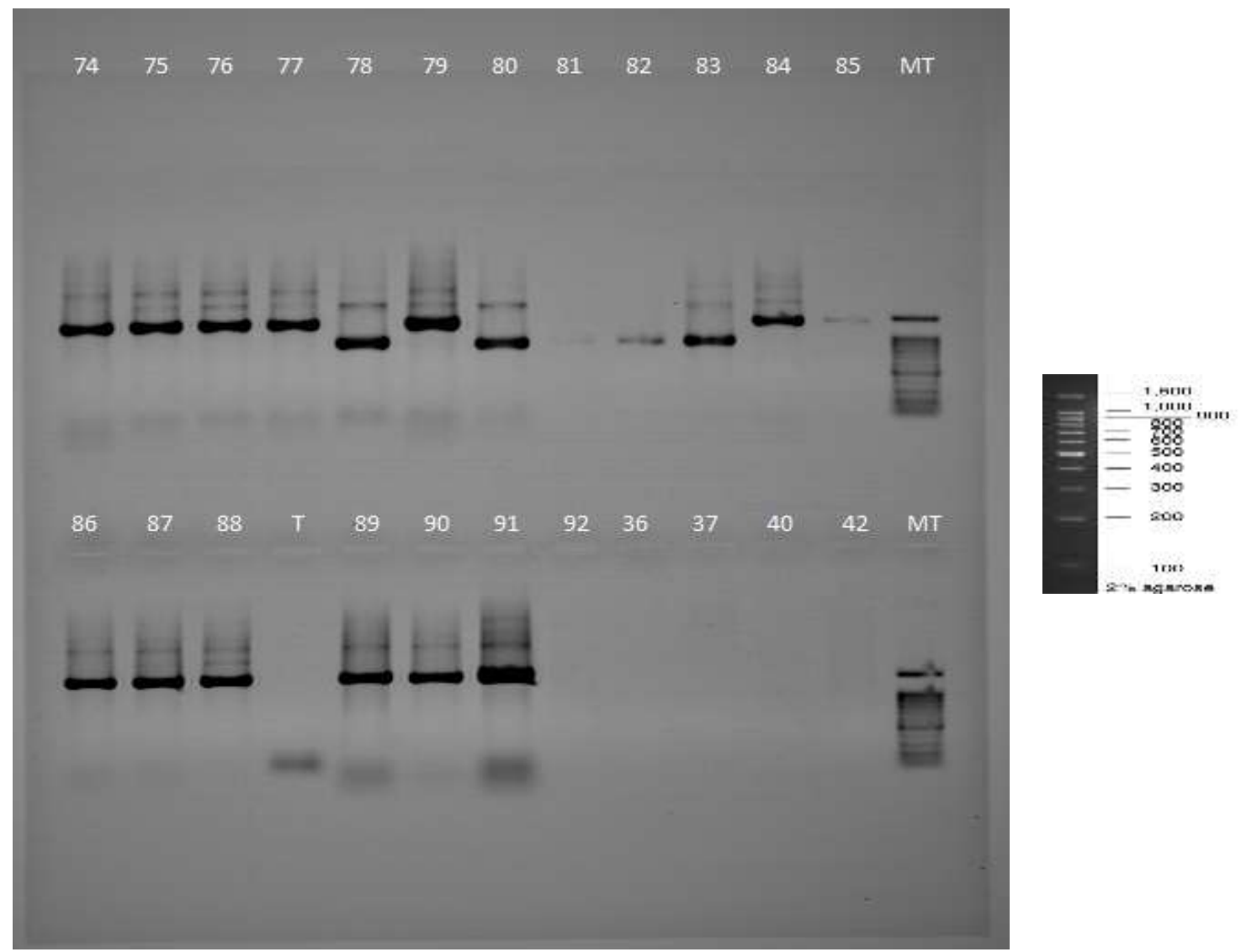

Figure 3:- Profil génétique de quelques accessions. (MT: marqueur de taille)

\section{Références:-}

1. AfricaRice. (2010) : New Breeding Directions at AfricaRice: Beyond NERICA. Cotonou, Benin: .24p. ISBN : 978-92-9113-340-6 (print)

2. Angeles-Shim, R. B., Asano, K., Takashi, T., Kitano, H., Ashikari, M. (2009): Mapping of the glabrous gene in rice using CSSLs derived from the cross Oryza sativasubsp. japonicacv. Koshihikari and O.glaberrima. Paper presented at the 6th International Rice Genetics Symposium, Manila, Philippines, 16-19 November.

3. Anonyme. (2002) : Mémento de l'agronome, Ministère des affaires étrangères - CIRAD -GRET; 799 - 811 (1687 p).

4. Carney, J. (2001): African rice in the columbian exchange. The Journal of African History., (42): 377-396.

5. Dally, A. M. and Second, G. (1990): Chloroplast DNA diversity in wild and cultivated species of rice (Genus Oryza, Section Oryza). Caldistic-mutation and genetic-distance analysis. Theor Appl Genet., $80: 209-222$.

6. Doyle, J. J and Doyle, J. L. (1987): A rapid isolation procedure for small quantities of fresh leaf tissue. Phytochem. Bul., 19: 11-15.

7. Garavito, A., Guyot, R., Lozano, J., Gavory, F., Samain, S., Panaud, O., Tohme, J., Ghesquière, A., Lorieux, M. (2010): A genetic model for the female sterility barrier between Asian and African cultivated rice species. Genetics.,185(4) : 1425-1440.

8. Gridley, H., Jones, M., Wopereis-Pura, M. (2002) : Development of New Rice for Africa (Nerica) and participatory varietal selection. In Witcombe, J., Parr, L. and Atlin, G. (eds.) Breeding rainfed rice for droughtprone environments: integrating conventional and participatory plant breeding in South and Southeast Asia. Gwynedd, Centre for Arid Zone Studies, University of Wales, pp.23-28.

9. Guyot, R., Garavito, A., Gavory, F., Samain, S., Tohme, J., Ghesquière, A., Lorieux, M. (2011): Patterns of sequence divergence and evolution of the s-1 orthologous regions between Asian and African cultivated rice species. Plos One., 6(3) : p. 17726. doi: 10.1371/journal.pone.0017726.

10. Hien, N. L., Sarhadi, W. A., Oikawa, Y., Hirata, Y. (2007) : Genetic diversity of morphological responses and the relationships among Asia aromatic rice (O. Sativa L.) cultivars. Topics., 16(4) : $13 \mathrm{p}$. 
11. Hiroaki, S. and Takeshi, I. (2010): Massive gene losses in Asian cultivated rice unveiled by comparative genome analysis. BMC Genomics., 11:121 http://www.biomedcentral.com/ 1471-2164/11/121, doi:10.1186/1471-2164-11-121

12. Huang, X., Kurata, N., Wei, X., Wang, Z. X., Wang, A., Zhao, Q., and al. (2012) : A map of rice genome variation reveals the origin of cultivated rice. Nature.,490:497-501.doi:10.1038/nature11532

13. Koide, Y., Ikenaga, M., Shinya, Y., Matsubara, K., Sano, Y. (2008) : Two loosely linked genes controlling the female specificity for cross-incompatibility in rice. Euphytica.,164(3) : 753-760.

14. Li, Z. M., Zheng, X. M. and Ge, S. (2011) : «Genetic diversity and domestication history of African rice (Oryza glaberrima) as inferred from multiple gene sequences». Theor. Appl. Genet., $123:$ pp. 21-31.

15. Mendez Del Villar, P., and Bauer, J. M. (2013) : Le riz en Afrique de l'Ouest: dynamiques, politiques et perspectives. Cah Agric., (22) : 336-44 doi : 10.1684/a9r.2013.0657.

16. Montcho, D., Gbénou, P., Missihoun, A. A., Futakuchi, K., Ahanhanzo, C., Agbangla, C. (2017) : Comparative study of two rice cultivars (Oryza glaberrima and Oryza sativa) under different cultural conditions, Journal of Experimental Biology and Agricultural Sciences., 5(1): 2320 - 8694, http://www.jebas.org. ISSN No.

17. Moukoumbi, Y. D., Sie, M., Vodouhe, R., Ogunbayo, S. A., N'dri, B., Toulou, B., Ahanchede, A. (2011): Assessing phenotypic diversity of interspecific rice varieties using agro-morphological characterization. J. Plant Breed. Crop Sci., 3: 74-86.

18. Murray, S. S. (2004) : Searching for the origins of African rice domestication. Antiquity 78p

19. Nabholz, B., Gautier, S., Sabot, F., Ruiz, M., Adam, H., Nidelet, S., Ghesquière, A., Santoni, S., David, J. and Glémin, S. (2014) : Transcriptome population genomics reveals severe bottleneck and domestication cost in the African rice (Oryza glaberrima). Molecular Ecology., 23 : 2210-2227.

20. Naoki, H., Naomi, M., Takayuki, K., Kazuhiro, U. and Ken I. (2010) : MPertohotdoolocgoy l: a simple gel-free method for SNP genotyping using allele-specific primers in rice and other plant species. Plant Methods., 6:12. http://www.plantmethods.com/content/6/1/12

21. Nayar, N. M. (2012): Evolution of the African Rice : A Historical and Biological Perspective. Crop Science Society of America., Vol 52, pp. 505 - 516.

22. Nokoe, S. (2001): Augmented block Design (ABD): the choice design for large scale farmer participatory onfarm trials, 5 p. (http : // www. Unu.edu/enu/plec).

23. Nwilene, F. E., Williams, U. T., Ukwangwu, M. N., Dakouo, D., Nacro, S., Hmadoun, A. and Kamara, S. I. (2002) : Reactions of differential genotypes to African gall midge in West Africa. Intel. Pest Manag.,48 : 195201.

24. Ojo, D. K., Ogunbayo, S. A., Sanni, A. K., Guei, R. G. (2009) : The determination of diversity and relationships among forty rice (Oryza sativa L.) accessions by comparative analysis of morphological and RAPD data 12p.

25. Orjuela, J., Sabot, F., Cheron, S., Vigouroux, Y., Adam, H., Chrestin, H.,Sanni, K., Lorieux, M. and Ghesquiere, A. (2014): An extensive analysis of the African rice genetic diversity through a global genotyping. Theor. Appl. Genet., 127(10): 2211 -2223.

26. Paterson, A. H., Bowers, J. E., Bruggmann, R. and al. (2009): The Sorghum bicolor genome and the diversification of grasses. Nature., $457: 551-556$.

27. Sano, Y. (1986): Sterility barriers between Oryza sativa and O. glaberrima. In: Rice Genetics. Proceedings of the International Rice Genetics Symposium, 27-31 May 1985. International Rice Research Institute, Manila, Philippines, pp. 109-118.

28. Sano, Y. (1990): The genic nature of gamete eliminator in rice. Genetics., 125: 183-191.

29. Sharma, J. R. (1988): Statistical and biometrical techniques in plant Breeding. New Age International Publishers. Edition 1988, Reprint 2006. 365p.

30. Tanksley, S. D., McCouch, S. R. (1997): Seed banks and molecular maps: Unlocking genetic potential from the wild. Science., 277: 1063-1066.

31. Vaughan, D. A., Lu, B. R. and Tomooka, N. (2008) : The evolving story of rice evolution. Plant Sci., $174: 394-$ 408.

32. Vaughan, D. A., Morishima, H. and Kadowaki, K. (2003) : Diversity in the Oryza genus. Current Opinion in Plant Molecular Biology., 6 : 139-146.

33. Vido, A. A. (2011): Le riz africain (Oryza glaberrima Steudel), un aspect de l'histoire rurale de la Côte de l'Or ou l'extension de la zone de « civilisation du riz » (XVIIè-XVIIIè Siècles). Revue Ivoirienne d'histoire, $\mathrm{N}^{\circ}$ 19, pp. $41-60$.

34. Xianneng, D., Jiawu, Z., Peng, X., Jing, L., Fengyi, H. and Dayun, T. (2010): The role of S1-g allele from Oryza glaberrima in improving interspecific hybrid sterility between $O$. sativa and $O$. glaberrima. Breeding Science 60: 342-346 doi:10.1270/jsbbs.60.342. 